\title{
Las hechuras del Conde Duque de Olivares. La alta administración de la monarquía desde el análisis de redes ${ }^{1}$
}

\author{
Francisco Gil MARTínEZ \\ Universidad de Almería \\ franciscogil@ual.es
}

Recibido: 22 de octubre de 2013

Aceptado: 9 de octubre de 2014

RESUMEN

El artículo aborda el estudio de la alta administración durante el valimiento del Conde Duque de Olivares utilizando como base los miembros de los Consejos y de las Juntas creadas durante este período. Para ello se aplican metodologías del análisis de redes a través de las cuales se aclara el papel de las Juntas estableciendo una clasificación de las mismas. Por último, se trata de identificar quiénes fueron los hombres que controlaron la administración durante este periodo y llevaron a la práctica las políticas dictadas por Olivares.

Palabras clave: Olivares, Juntas, Consejos, Consejeros, redes.

\section{The Minions of the Count Duke of Olivares. Social Network Analysis of the Monarchy's Bureaucracy}

\begin{abstract}
The article studies the high administration during the influence of the Count Duke of Olivares using the members of the Councils and the Juntas created in that period. We use methodologies of network analysis to clear the role played by the Juntas, and we also make a classification of them. Finally, we identify the men who controlled the administration at that time and implemented Olivares' politics.
\end{abstract}

Key words: Olivares, Juntas, Councils, Councilors, Networks.

1 El presente estudio se ha realizado en el marco del Proyecto de Investigación de I+D El poder del dinero. Dimensiones de la venalidad en los siglos XVII y XVIII (HAR2011-23105) financiado por el Ministerio de Economía y Competitividad. 
Durante el valimiento de Gaspar de Guzmán, el recurso a las Juntas como mecanismo administrativo, lejos de ser un medio extraordinario se tornó en un auténtico método de gobierno, llegando a constituir nuevas vías administrativas que venían a sumarse a las de los tradicionales Consejos. La creación de estas instituciones estuvo vinculada a las ideas reformistas del valido de Felipe IV, pues muchas de ellas eran la plasmación institucional de novedades fiscales, como la Junta de la Media Annata o la del Papel Sellado, mientras que otras trataban de racionalizar la gestión de ciertos asuntos fundamentales, como la Junta de Presidios o la Junta de Minas. Estas Juntas fueron erigidas sin una legislación clara que sancionase sus competencias y funcionamiento. Contamos con varios estudios sobre Juntas concretas ${ }^{2}$, como es el caso de la Junta del Almirantazgo, estudiada por Alloza Aparicio ${ }^{3}$, o la Junta de Media Annata, estudiada por Cárceles de $\mathrm{Gea}^{4}$. Sin embargo, apenas tenemos datos de muchas de ellas $^{5}$, carencia en parte motivada por la escasez de documentación ${ }^{6}$. Las Juntas han sido objeto de interés especial por parte de los investigadores especialistas en Historia del Derecho. Hace muchos años que Espejo Hinojosa inició esta línea de trabajo desde una óptica general, entendiendo las Juntas como un sistema institucional ${ }^{7}$, pero

2 Entre estos estudios hemos de citar García-Badell Arias, L. M.: "La Junta Grande de Competencias de Felipe IV: Rey, nobleza y Consejos en la Monarquía Católica", Cuadernos de Historia del Derecho, Extraordin (2004), pp. 105-136; Jiménez Moreno, A.: "Honores a cambio de soldados, la concesión de hábitos de las Órdenes Militares en una coyuntura crítica: la Junta de Hábitos (1635-1642)", en Soria MesA, E. y MolinA Recio, R. (eds.): Las élites en la época moderna: la Monarquía Española. Volumen III: Economía y poder, Córdoba, Universidad de Córdoba, 2009, pp. 155-17; BALtar RodríGuEZ, J. F.: "Sobre el origen de la Junta de Guerra de Indias", en GonzÁlez Vale, L. (ed.): Actas y Estudios del XIII Congreso del Instituto Internacional de Historia del Derecho Indiano, San Juan, Academia Puertorriqueña de la Historia, 2003, pp. 671-686; DíAZ GonzÁlez, F. J.: La Real Junta de Obras y Bosques en la época de los Austrias, Madrid, Dykinson, 2002; Domínguez Nafría, J. C.: "La Junta de Guerra de Indias", Temas de Historia Militar: $2^{\circ}$ Congreso de Historia Militar, Zaragoza, 1988, pp.79-116; LABrador Arroyo, F.: "El gasto y la financiación de las obras y oficiales de los bosques y sitios reales de Castilla entre 1609 y 1625", en el XXXI Encontro Aphes, Coimbra, 2011, pp. 1-18; González Palencia, A.: La Junta de Reformación. Documentos procedentes del Archivo Histórico Nacional y del General de Simancas, Valladolid, Archivo Histórico Español, 1932.

3 Alloza Aparicio, A.: "La Junta del Almirantazgo y la lucha contra el contrabando, 1625-1643”, Espacio, 16 (2003), pp. 217-254. También sobre la Junta de Almirantazgo DíAz GonzÁlez, F. J.: "Los miembros de la Real Junta del Almirantazgo", Historia, instituciones, documentos, 26 (1999), pp. 193-210.

4 CÁRCELES DE GEA, B.: "La junta de la media annata: presión fiscal y honor en el siglo XVII castellano", Cuadernos de investigación histórica, 15 (1994), pp. 177-192.

5 En estudios sobre el periodo podemos encontrar datos referentes a la actividad de alguna de ellas como la Junta de Coronelías en Jiménez Estrella, A.: "El reclutamiento en la primera mitad del XVII y sus posibilidades venales", en Andúuar Castillo, F. y Felices de la Fuente, M. M. (eds.): El poder del dinero: Ventas de cargos y honores en el Antiguo Régimen, Madrid, Biblioteca Nueva, 2011, pp. 169-190; y en RoDRíGUEZ HERnÁndeZ, A. J.: "Servir al rey con hombres. Recompensas concedidas a élites y representantes del rey por su colaboración en el reclutamiento (1630-1700)", en Esteban EstrínganA, A. (ed.): Servir al rey en la Monarquía de los Austrias. Medios fines y logros del servicio al soberano en los siglos XVI y XVII, Madrid, Sílex, 2012, pp. 415-443. O las Juntas relativas al escuadrón de la nobleza estudiadas por JimÉnEz MorEnO, A.: "Las repercusiones de la guerra sobre los privilegiados y la asistencia al monarca: el escuadrón de la nobleza (16411643)", Estudios Humanísticos. Historia, 10 (2011), pp. 71-94.

6 En los archivos españoles hay documentación de unas pocas Juntas, por lo que se supone que el grueso de estas acabaron en el archivo personal del Conde Duque, que se perdió en su mayoría. Cfr. ElLiOTT, J. H.: El Conde-Duque de Olivares: el político en una época de decadencia, Barcelona, Crítica, 1998, p. 734.

7 Espejo Hinojosa, C.: "Enumeración y atribuciones de algunas juntas de la administración española desde el siglo XVI hasta el año 1800”, Revista de la Biblioteca, Archivo y Museo de la Comunidad de Madrid, 32 (1931), pp. 325-362. 
el campo permaneció prácticamente inexplorado hasta que Tomás y Valiente dio los primeros pasos para contextualizar las Juntas dentro del organigrama administrativo del Estado ${ }^{8}$. Siguió esta línea José Luis Bercuyo ${ }^{9}$ y tras él, los dos autores que más han profundizado en el tema hasta la fecha, también historiadores del derecho: Dolores Sánchez González y Juan Francisco Baltar Rodríguez, siendo este último quien con más extensión se ha ocupado de la situación jurídica de las Juntas y sus relaciones con el resto de las instituciones. Las publicaciones de ambos son el producto de sus respectivas tesis doctorales y presentan varias similitudes, principalmente en el enfoque que dan al tema al profundizar ambos en la situación jurídica de las Juntas y en las relaciones de estas con el resto de instituciones.

En primer lugar, hemos de aclarar el uso del término "Junta" que empleamos, pues su polisemia puede dar lugar a equívocos. Según recoge el diccionario de autoridades de 1734 el término "Junta" se definía como "Ayuntamiento o congreso de varias personas en un mismo lugar para consultar y resolver alguna materia". El empleo de esta acepción en la documentación de la época, ha creado confusión entre los historiadores. Por un lado, la voz "Junta" se refería a las comisiones de ministros para un asunto concreto y, por otro, a los órganos colegiados cuya finalidad era gestionar un tema que se preveía persistente en el tiempo. Dolores Sánchez ha diferenciado entre Juntas extraordinarias o "ad hoc", si no tenían vocación de permanencia, y Juntas ordinarias, si gozaban de una competencias y estructura estables ${ }^{10}$. Concretando en un ejemplo esta distinción teórica, la "Junta para debatir la introducción del consulado y Lonja en la Corte" de $1634^{11}$ fue una de las Juntas extraordinarias, mientras que la Junta de Media Annata se insertó en la estructura de la administración de forma estable, y sería, por tanto, una Junta ordinaria. Es este último tipo de Juntas el objeto de estudio del presente trabajo y al cual nos referiremos a partir de ahora, pues abarcar todas las Juntas extraordinarias que hubo durante el periodo de Olivares sería imposible en el marco de esta aportación.

Para entender el fenómeno de las Juntas creemos necesario que su estudio corra parejo con el de los Consejos, por varios motivos. El primero de ellos es que las Juntas presentan la misma estructura que los Consejos, pues estaban constituidas por un presidente, varios consejeros y el personal administrativo, pero a una escala reducida, y con una diferencia significativa, y es que carecían de fiscales. Este elemento nos indica claramente el mayor control que tuvo el soberano, o su valido, sobre las Juntas en comparación con los Consejos, puesto que no incluyeron fiscales que las controlasen. En segundo lugar, las competencias de las Juntas se situaban en el ámbito de los Consejos, por lo que cambiaron de forma radical el equilibrio de poderes en la alta administración. Y, por último, las Juntas estuvieron en parte compuestas por el

8 Tomás y VAliente, F.: El gobierno de la monarquía y la administración de los reinos en la españa del siglo XVII", en Tomás y VALIEnTE, F. (ed.): La España de Felipe IV : el gobierno de la monarquía, la crisis de 1640 y el fracaso de la hegemonía europea, Madrid, Espasa Calpe, 1982, pp. 3-214.

9 Bercuyo, J. L.: «Notas sobre Juntas del Antiguo Régimen», en Actas del IV Symposium de Historia de la Administración, Madrid, Instituto Nacional de Administración Pública, 1983, pp. 93-108.

10 SÁnchez González, D. M.: Las Juntas Ordinarias. Tribunales permanentes en la corte de los Austrias, Madrid, UNED, 1995.

11 Baltar Rodríguez, J. F.: Las juntas de gobierno de la monarquía hispánica: (siglo XVI-XVII), Madrid, Centro de Estudios Políticos y Constitucionales, 1998, p. 160. 
mismo personal que los Consejos. Ignorar estos elementos supone realizar un estudio de las Juntas totalmente descontextualizado.

Hasta ahora, la alta administración ha sido estudiada a través de las leyes que la regulaban, estudios de instituciones concretas o mediante el cursus honorum de los consejeros, siendo el mejor ejemplo el estudio de Janine Fayard sobre los miembros del Consejo de Castilla ${ }^{12}$. La alta administración de los Habsburgo ha sido siempre estudiada desde la perspectiva del individuo, ya sea desde dentro o desde fuera de la institución o mediante los reglamentos que regulaban las instituciones. Los conocimientos que ya tenemos gracias a estas vías de estudio y las metodologías adoptadas de otras disciplinas científicas nos permiten plantear nuevas líneas de trabajo. El principal punto que planteamos en este trabajo es el cambio de óptica, pasar del individuo y la institución a estudiar de forma conjunta la alta administración y el papel que jugaron las Juntas en este sistema. Nuestro objeto de estudio son los Consejos y Juntas como componentes de un sistema y no como un conjunto de instituciones agregadas. Así, trataremos de identificar a los hombres que controlaron la administración durante este periodo.

Generalmente tanto la teoría de redes como la de grafos se han empleado para estudiar sistemas de individuos y las relaciones que éstos establecen entre sí. En este trabajo hemos utilizado esta perspectiva partiendo de la base de que dos individuos que forman parte de una misma institución mantienen entre sí una relación. Dos individuos que se reúnen todas las semanas en una determinada institución forzosamente han de conocerse, aunque no entramos a valorar los términos de esa relación. En este caso no es una relación familiar, de amistad o de negocios, se trata de una relación de trabajo. Además de las relaciones entre consejeros tratamos las relaciones de las instituciones entre sí, que unas veces fueron de colaboración y otras de rivalidad. Entendemos que un individuo que forma parte de dos instituciones al mismo tiempo constituye, en sí mismo, un canal privilegiado de comunicación entre éstas. La acumulación de nombramientos en un individuo, lejos de ser una excepción, fue una práctica muy frecuente durante el valimiento del Conde Duque, creando vías de comunicación entre todas las instituciones de la alta administración. Esto nos permite estudiar las instituciones con la misma metodología empleada para los individuos, observando las relaciones que se establecen entre ellas mediante los miembros comunes, visión opuesta a la anterior pero complementaria de la misma.

En este trabajo empleamos metodologías que ya se utilizan en otras disciplinas, adaptándolas al estudio de las instituciones a lo largo de la Historia, empleando técnicas matemáticas, de uso frecuente en sociología, como son las operaciones con matrices, el análisis de redes y la teoría de grafos. La utilidad de estas técnicas en historia ha sido probada en numerosos trabajos de historia social, sobre grupos de individuos y sus relaciones personales ${ }^{13}$. No obstante, aún no se han explotado todas las posibilidades de estas herramientas debidamente aplicadas desde una perspectiva crítica.

12 FAYARD, J.: Los miembros del Consejo de Castilla (1621-1746), Madrid, Siglo XXI, 1982.

13 Un estado de la cuestión sobre redes sociales e historia así como diversos trabajos con una sólida base teórica se encuentran en el monográfico coordinado por Michel Bertrand, Sandro Guzzi y Claire Lemercier en REDES Revista hispana para el análisis de redes sociales, 21 (2011). Como trabajo pionero destacamos el de SÁnchez Balmaseda, M. I.: Análisis de redes sociales e historia, una metodología para el estudio de redes 
Para este trabajo, partimos de los individuos que formaban 18 de las Juntas que estuvieron activas entre 1635 y 1640. Los datos referentes a los miembros los hemos obtenido de la obra de Baltar Rodríguez y los hemos contrastado con los estudios monográficos que existen para alguna de estas Juntas citados anteriormente. Las Juntas estudiadas son las siguientes: Minas, Población de Madrid, Almirantazgo, las diversas Juntas del Donativo ${ }^{14}$, Media Annata, Defensa de los Reinos, Presidios, Coronelías, Artillería, Hábitos, Reclutamiento, Papel Sellado, Ejecución, Nobleza y la de Vestir la Casa. Hemos incluido el Consejo de la Sal y la Comisión de Millones ${ }^{15}$ como Juntas porque ambas instituciones son frecuentemente denominados como Junta y porque ocupan una posición administrativa y jurídica análoga a la del resto de Juntas. También se encuentran presentes en el estudio las salas que se originaron tras la disolución de la Junta de Ejecución, pues venían a asumir sus funciones. Para los Consejos hemos tomado como base un artículo de Quintín Aldea en el que reconstruye la composición de todos los Consejos en el año $1635^{16}$ y cuya fiabilidad hemos contrastado comparándola con los miembros que citan las obras monográficas sobre los diferentes Consejos.

En total analizamos 147 individuos y 30 instituciones diferentes. Para trabajar con estos datos hemos construido una matriz, en la que hemos situado a los consejeros en las filas y a las instituciones en las columnas ${ }^{17}$. Los gráficos que mostramos han sido realizados mediante los programas de software Ucinet y Netdraw, a partir de esa matriz. En ellos se representan los diferentes nodos o actores, como formas geométricas, unidas por líneas, que indican la existencia de una relación entre ellos. La leyenda de todos los gráficos se encuentra en el anexo donde se incluyen los datos que hemos manejado. Las operaciones que aquí hacemos son sólo una muestra del potencial que tienen estos programas de software aplicados al campo de la historia ${ }^{18}$.

\section{UNA PROPUESTA DE CLASIFICACIÓN DE LAS JUNTAS}

La posición jurídica de las Juntas en el entramado administrativo ha sido el objeto fundamental de los principales estudios generales sobre ellas, sin embargo, no fue una característica identificativa de las mismas. Hubo tantas situaciones jurídicas diferentes como Juntas, como ya han probado los trabajos a los que hemos hecho refe-

clientelares, Madrid, Universidad Complutense, 2002. Para una bibliografía menos técnica remitimos a PONCE Leiva, P. y Amadori, A.: "Redes sociales y ejercicio de poder en la América Hispana: consideraciones teóricas y propuestas de análisis", Revista Complutense de Historia de América, 34 (2008), pp. 15-42.

14 En este caso lo hemos contemplado como una única institución, pues fueron idénticas con la diferencia del año del donativo al que se dedicaban.

15 CÁrceles de GeA, B.: Fraude y administración fiscal en Castilla. La Comisión de Millones (16321658). Poder fiscal y privilegio jurídico-político, Madrid, Banco de España, 1994.

16 Aldea Vaquero, Q.: "Los miembros de todos los Consejos de España en la década de 1630 a $1640 "$, Anuario de historia del derecho español, 50 (1980), pp. 189-205.

17 Un 1 en una celda indica que el individuo de la fila correspondiente es consejero de la institución que encabeza la columna, mientras que un 0 representa la ausencia de relación entre ambos. Es importante que todas las celdas sean completadas de esta forma para las operaciones posteriores.

18 Para profundizar en esta metodología y su aplicación a la historia resulta interesante aunque algo desfasada la tesis de SÁnchez Balmaseda, op. cit. (nota 13). 
rencia. En ellos, aparte de la clasificación entre Juntas ordinarias y extraordinarias, se establece como principal criterio el temático. Basándonos en estos estudios y en la metodología de análisis que aquí planteamos, proponemos una nueva clasificación de las mismas en base a sus competencias y el origen de sus miembros. Por tanto, hemos distinguido entre Juntas con un carácter político y otras enfocadas a cuestiones de carácter técnico. Las primeras, generalmente entraban en conflicto con los Consejos al suplantarlos en la toma de decisiones de especial trascendencia, mientras que las de carácter técnico se ocupaban de temas concretos de menor importancia, como la puesta en marcha de una determinada medida, que requerían de unos conocimientos técnicos y generaban una gran cantidad de trabajo.

Esta primera clasificación en base a las atribuciones de las Juntas está estrechamente relacionada con dos perfiles diferentes de consejero. Las de carácter político estaban formadas principalmente por cargos importantes del entorno cortesano, entre los que se encontraban numerosos parientes del propio Conde Duque, miembros a su vez del Consejo de Estado. Estas Juntas sí que se ajustan, en mayor o menor medida, a la definición que la literatura clásica ha dado sobre las Juntas en general que eran las empleadas por Olivares para tratar con sus más cercanos aliados los temas más importantes de la política interior y exterior ${ }^{19}$. Estas relaciones personales fueron la única defensa frente a las instituciones tradicionales, por lo que una vez desaparecido Olivares de la escena política cayó también todo ese entramado político-administrativo. Ejemplo de Junta de carácter político fue la polémica Junta de Ejecución, que se creó como un canal privilegiado para tratar asuntos urgentes de cierta importancia ${ }^{20}$. La indeterminación de sus atribuciones dio un amplio margen de gobierno a quienes la formaban. No es de extrañar que el Consejo de Castilla fuese quien liderase el ataque hacia esta Junta al verse relegado por las amplias facultades conferidas a la Junta de Ejecución ${ }^{21}$.

Por otra parte, encontramos otras Juntas con un carácter mucho más técnico, con atribuciones pertenecientes a distintos Consejos y que requerían, según el criterio del Conde Duque, una atención más especializada. Estaban formadas por consejeros que, en su mayoría, eran burócratas de carrera sin oficios cortesanos. Estas Juntas suscitaron una oposición mucho menor entre los Consejos y muchas de ellas sobrevivieron a la caída de Olivares. Un ejemplo paradigmático de esta segunda tipología de Juntas fue la Junta de Minas, que recogía las competencias referentes a la gestión de las minas de todos los territorios de la Monarquía, aunque por su distribución geográfica se centraba en las de Indias y Castilla, por lo que contaba como vocales con consejeros de ambas instituciones. Otro ejemplo de Junta de carácter técnico y de jurisdicción interterritorial fue el de la Junta del Almirantazgo ${ }^{22}$, dedicada a una cuestión internacional que implicaba a varios territorios de la Monarquía como lo era evitar el contrabando con las Provincias Unidas. Esta Junta fue absorbida por el Consejo de Guerra en 1643, pues su función estaba estrechamente vinculada a la política internacional.

19 Elliott, op. cit. (nota 6) p. 335.

20 Baltar RodríGUEZ, op. cit. (nota 11), p. 394.

21 Ibidem, p. 401.

22 Para esta Junta contamos con el estudio de Alloza Aparicio, op. cit. (nota 3). 
Mientras que las Juntas de carácter político trataban de restar competencias a los Consejos y hacer que la toma de decisiones recayese en oficios muy vinculados a la Corte, las de carácter técnico cumplieron un papel diferente. Resulta imprescindible la referencia al trabajo de Cárceles Gea sobre la Junta de Media Annata ${ }^{23}$, cuya tesis se basa en que su creación permitía gestionar un derecho a cobrar en todos los territorios de la Monarquía, algo que no era posible realizar mediante el Consejo de Hacienda pues tenía limitada su jurisdicción a los territorios de la Corona de Castilla. Similar función cumplió la Junta del Papel Sellado ${ }^{24}$ en la introducción de este arbitrio. La efectividad de ambas las hizo sobrevivir, más o menos supeditadas al Consejo de Hacienda, tras el cambio de gobierno que supuso la caída en desgracia del Conde Duque de Olivares, algo que no sucedió con las Juntas más conocidas del reinado que fueron sin duda las de carácter político.

En definitiva, las Juntas políticas trataban de sustituir a los principales órganos en la toma de decisiones, mientras que las Juntas de carácter técnico incrementaron el aparato burocrático para absorber el volumen de trabajo generado por los conflictos bélicos, los nuevos derechos a percibir por la corona y otras políticas concretas. No debemos olvidar que el establecimiento de estos nuevos organismos conllevaba la creación de nuevas plazas de secretarios, tesoreros, escribanos, y demás oficiales, necesarios para gestionar una creciente cantidad de trabajo administrativo.

\section{ANÁLISIS DE REDES}

En este trabajo hemos incluido varias representaciones gráficas de los datos, cuando hemos considerado que aportan claridad a la exposición. En los casos en que los gráficos resultantes eran demasiado complejos para ser interpretados, hemos recurrido a otras técnicas que detallamos más adelante.

En el gráfico 1 aparecen representados los diferentes Consejos de la Monarquía junto con sus miembros. Como se puede apreciar, todos los Consejos compartían al menos uno de sus miembros con otro Consejo, lo que hacía que todos estuviesen vinculados entre sí, pues como vemos se trata de un grafo con todos sus nodos conectados. Para 1635, ninguno de los Consejos contaba con la dedicación exclusiva de todos sus miembros. Se conoce el cursus honorum de los ministros a través de los Consejos según la importancia de éstos, pero apenas se ha prestado atención al hecho de que muchos de estos ministros, que desempeñaban cargos en más de un Consejo, pudieran ser una pieza clave en la coordinación de todo el sistema administrativo. Sería interesante un estudio más profundo, y cronológicamente mucho más amplio, para conocer si esa fue una característica coyuntural o si se extendió durante todo el Antiguo Régimen.

23 Cárceles de Gea, op. cit. (nota 4).

24 Baltar Rodríguez, J. F.: "Notas sobre la introducción y desarrollo de la renta del papel sellado en la Monarquía Española (siglos XVII y XVIII)”, Anuario de historia del derecho español, 66 (1996), pp. 519-560. 


\section{Gráfico 1}

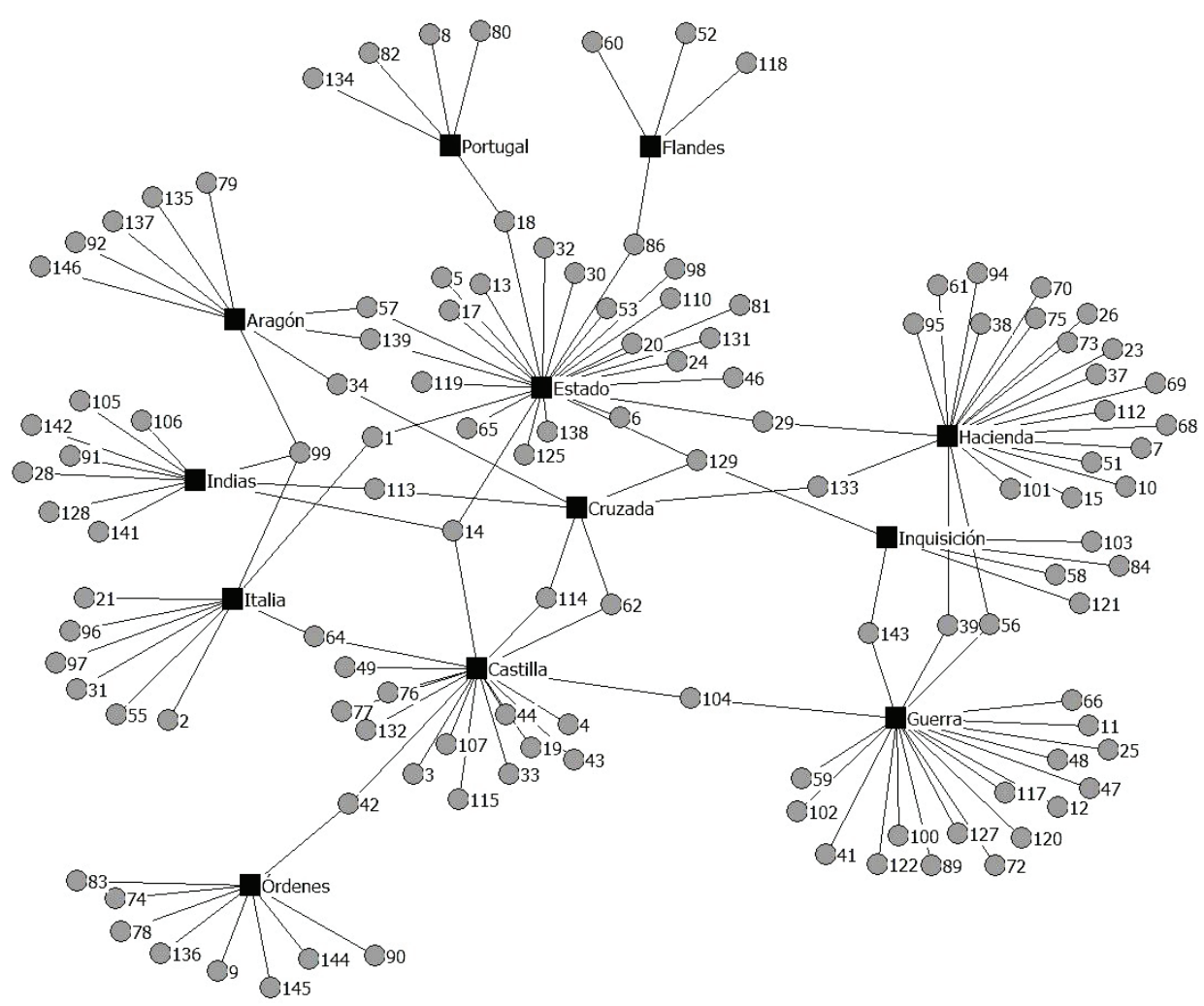

Entrando en un análisis pormenorizado, encontramos un número mayor de individuos que se relacionan con una única institución que los que lo hacen con varias. O dicho de otra manera, los Consejos, a pesar de no contar con la dedicación exclusiva de todo su personal, solo compartían a unos pocos consejeros con otros Consejos. La distribución de los individuos en torno a las instituciones en forma de estrella o abanico, es una característica típica de las redes de máxima centralización, pues no hay otras relaciones que produzcan distorsiones en la posición. En cambio, los actores que participan en dos o más Consejos se ven representados en los espacios intermedios entre Consejos, separados para mantener una equidistancia entre las dos instituciones a las que pertenecían. Con estos criterios, hemos centrado nuestra atención sobre aquellos pocos individuos, en relación al total, que formaban parte de varios Consejos. Las vías de comunicación interinstitucional que constituían estos intermediarios ayudaban a mantener la cohesión de todo el sistema, además de ejercer un posible control del mismo desde una posición privilegiada. Por tanto, no es de extrañar que fuesen los colaboradores más cercanos del Conde Duque de Olivares, quienes ocupasen más de un asiento en los Consejos. Encontramos a sus propios familiares, como su cuñado, el conde de Castrillo (14), a personajes del ámbito cor- 
tesano como el Inquisidor General, Fray Antonio de Sotomayor ${ }^{25}$ (129) o el Factor General, Bartolomé Espínola ${ }^{26}(56)$, y también a personajes que hicieron carrera en la administración de la mano del valido, llegando hasta las más altas esferas, como fue el caso de José González ${ }^{27}$ (62).

Hemos de advertir sobre los datos indicados la facilidad de cometer errores al realizar un análisis de redes tal y como se hace en sociología, principalmente porque los Consejos no tenían la misma importancia. También los consejeros acaparaban poder por otras vías que no aparecen en este estudio, pues quedan fuera todos los cargos palaciegos y las relaciones clientelares que mantenían en el entorno cortesano. Ambas situaciones tienen un claro ejemplo en el Consejo de Cruzada, que aparece en una posición central en la red. Podríamos llegar a la conclusión de que el Consejo de mayor importancia era el de Cruzada pero, si recurrimos a cualquier estudio sobre los Consejos, descubrimos que el de Cruzada no solo carecía de la importancia que la representación le atribuye sino que, por el contrario, fue uno de los menos relevantes. Los nombramientos honorarios de consejeros son otro elemento que podría introducir distorsiones en la representación gráfica. Por este motivo, únicamente hemos considerado a los consejeros que formaron parte de las instituciones de forma efectiva, descartando los nombramientos honorarios que hemos localizado. Estos ejemplos son significativos de la necesidad de cotejar los resultados de varias metodologías, con el fin de corregir las deficiencias de cada una de ellas.

En el gráfico 2 se representa el sistema formado por las Juntas que hemos estudiado y sus miembros utilizando los mismos parámetros que en el anterior. Como se puede apreciar, las Juntas también formaban un sistema en el que todas están interrelacionadas. En este caso la densidad de la red es mayor y quedan más difuminados los espacios de cada una de ellas al existir más individuos que forman parte de varias Juntas. Para la comparación con el gráfico 2, hemos elaborado un índice que mide el promedio de las instituciones de las que formaba parte un individuo. Al aplicarlo a ambos casos, el promedio de Consejos en los que tenía plaza cada consejero, era de 1,21, mientras que, aplicado al sistema de Juntas, este mismo índice asciende a 1,82. Estos dos datos no tienen otra aplicación que la comparación de los dos sistemas, pero nos resultan útiles a la hora de cuantificar lo que muestran los gráficos. No hemos empleado las medidas de centralidad y densidad que se utilizan tradicionalmente en el estudio de redes en sociología porque manejamos dos tipos diferentes de nodos, para evitar así que se produzca una distorsión en esos índices.

En el caso de las Juntas, no solo era más frecuente que compartieran sus miembros, sino que se daban casos en los que un mismo consejero formaba parte en un momento dado de hasta siete de ellas, cuando en el ámbito de los Consejos no hubo ningún consejero que lo fuese en más de tres. Este primer análisis básico entre los dos tipos

25 Negredo del Cerro, F.: "Gobernar en la sombra. Fray Antonio de Sotomayor confesor de Felipe IV. Apuntes políticos”, Mágina, Revista Universitaria, 13 (2009), pp. 85-102.

26 Álvarez del Nogal, C.: "El factor general del Rey y las finanzas de la Monarquía Hispánica", Revista de Historia Económica, 3 (1999), pp. 507-539.

27 FaYARD, J.: "José González (1583?-1668), creatura du Comte-Duc d'Olivares, conseiller de Phillipe IV", en Duran, Y. R.: Homage a Roland Mousnier. Clientèles et fidélités en Europe a l'epoque moderne, París, Presses universitaires de France, 1981, pp. 351-368. 
de instituciones nos aporta un elemento muy interesante que nos ayuda a explicar la eclosión de Juntas, pues es indudable que estos nuevos organismos fomentaban, en mayor medida que los Consejos, la participación de sus miembros en otras instituciones. Esta interrelación permitía aumentar la facilidad con la que se transmitía la información en la administración, lo que repercutía a su vez en una mayor comodidad para controlar la red desde una posición central, pues existían más vías para comunicar con las distintas instituciones.

\section{Gráfico 2}

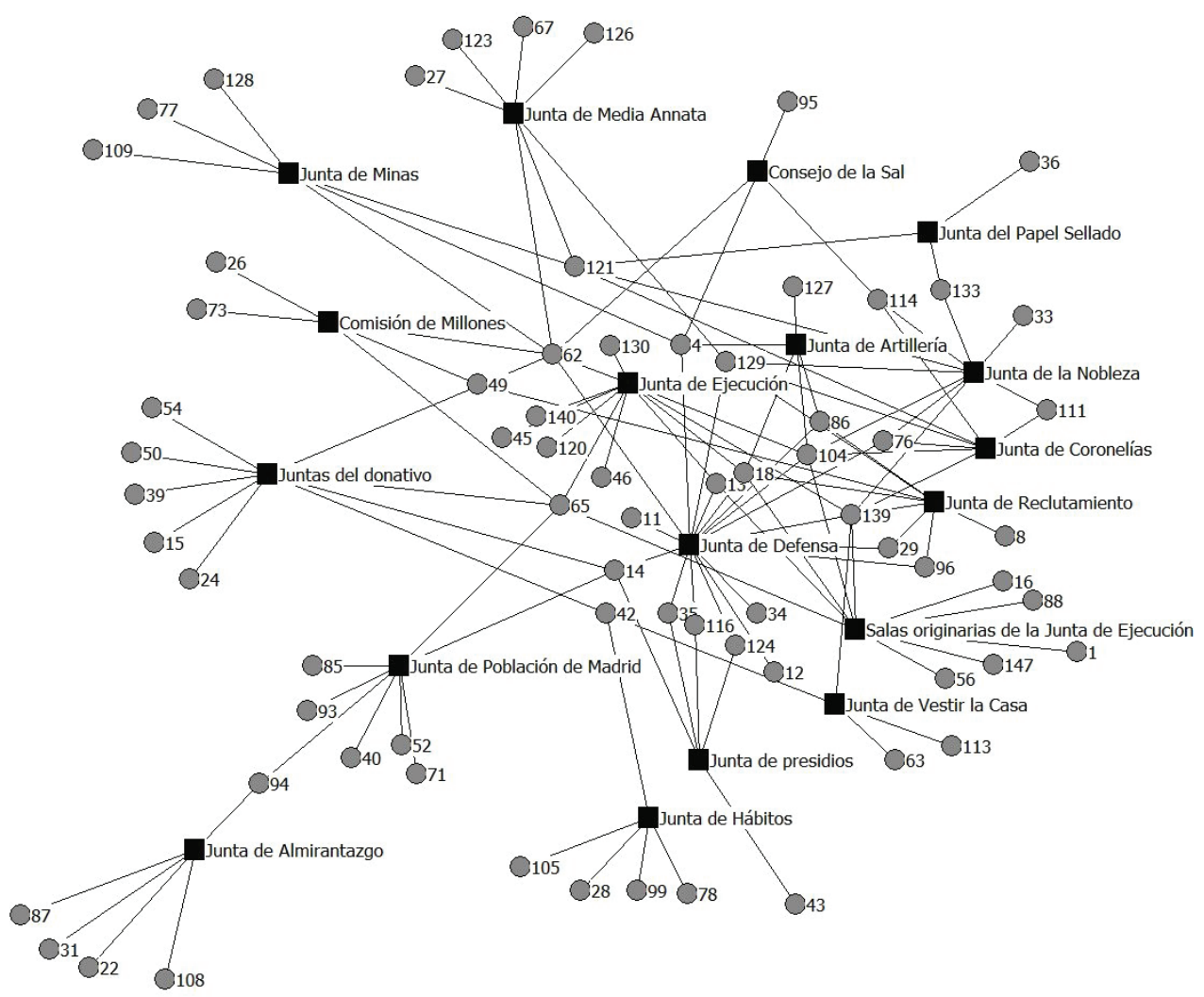

Como mencionamos al principio, las Juntas fueron otra expresión de la política reformista de Olivares. Las numerosas dificultades para reformar el sistema polisinodial llevaron al valido a utilizar mecanismos indirectos como el de las Juntas. La participación de sus miembros en diferentes Juntas y Consejos creaba nuevas relaciones entre las instituciones a través de sus miembros. De hecho, multiplicaba el número de conexiones, hasta el punto de que el índice de instituciones por individuo, para los miembros de Consejos y Juntas agregados, es de 1,86, superior a los dos anteriormente expuestos. La representación gráfica resultante en este caso presenta tal número de relaciones que hemos renunciado a incluirla en este trabajo por cuestión de claridad. 
Debido a las dificultades técnicas que plantea la compleja red resultante, hemos recurrido a herramientas que ayudan a simplificarla, como son los análisis de segundo nivel, que tienen la virtud de aclarar la representación gráfica, pues eliminan los nodos que sirven como enlace pero sin suprimir las conexiones que establecían. En este caso, al utilizar en nuestro trabajo dos tipos de nodos diferentes, instituciones y miembros, únicamente hemos aplicado este análisis a las instituciones, en tanto que para los individuos hemos empleado otro método más eficaz, dadas las particularidades de la red. El primer objetivo, era representar las relaciones entre instituciones eliminando de la representación a los miembros. Para ello partimos de una matriz simétrica en la cual, tanto columnas como filas, están ocupadas por las instituciones. Los números de las celdas de esta matriz representan la relación existente entre las instituciones de la columna y la fila correspondientes. Así un 3 en una celda significa que la institución de la fila y la de la columna tienen 3 miembros en común. Un grupo de celdas podría plantear algún problema de interpretación, pues las celdas situadas en la diagonal hacen referencia a la misma institución en la columna y en las filas. Sin embargo, estas celdas indican la relación de la institución consigo misma a través de sus miembros lo que, expresado en lenguaje normal, no es otra cosa que el número de miembros de esa institución.

Ahora bien, la confección de esta matriz simétrica podemos hacerla a través de los datos, buscando a qué instituciones pertenecía cada miembro y anotándolo en cada una de ellas después sumar el total de cada celda. Este método, aunque factible para matrices pequeñas o poco relacionadas, sin embargo, para las de mayor tamaño y relaciones supone una gran cantidad de operaciones, lo que multiplica la posibilidad de cometer errores. Resulta más práctico realizar una operación de cálculo matricial con un programa de hojas de cálculo como Open Office Calc o Excel. En la matriz original de la que hemos generado los gráficos 1 y 2, los miembros ocupaban las columnas y las instituciones las filas, y en las celdas únicamente había ceros y unos dependiendo de si el individuo pertenecía a la institución. Para obtener una matriz que nos permita hacer un análisis de segundo grado, únicamente debemos multiplicar esa matriz de origen por su traspuesta, es decir, la misma matriz pero intercambiando filas y columnas. El resultado será una matriz que representará las relaciones de segundo grado entre los elementos que se sitúen en las columnas de la matriz que se ubique en primer lugar. Si queremos calcular las relaciones de segundo grado de los elementos de las filas en lugar de los de las columnas de la matriz de origen, únicamente debemos cambiar el orden de la multiplicación poniendo en primer lugar la matriz traspuesta.

La representación de esa matriz de segundo grado es el gráfico 3, en el cual ya únicamente aparecen las instituciones y las relaciones que estas mantenían entre sí. A pesar de que la cantidad de relaciones hace difícil la lectura, podemos observar cómo se establece un núcleo muy interrelacionado del que participan los Consejos de Estado y Castilla y, en menor medida, el de Cruzada. En el gráfico, la mayoría de las Juntas están situadas en el centro de la red, ocupando los huecos entre Consejos y minimizando las líneas. Solo unas pocas, como la Junta de Almirantazgo o la Junta del Papel Sellado, tienen posiciones periféricas en la red. Por otro lado, en el gráfico se pueden distinguir entre Juntas políticas y técnicas en función de la posición de las 
mismas. En el centro, compitiendo por el poder, se encuentran las políticas, destacando la Junta de Ejecución, mientras que en la periferia del gráfico se encuentran las de carácter técnico, con unas relaciones mucho menos intensas con núcleos de poder como el Consejo de Estado o el de Castilla.

\section{Gráfico 3}

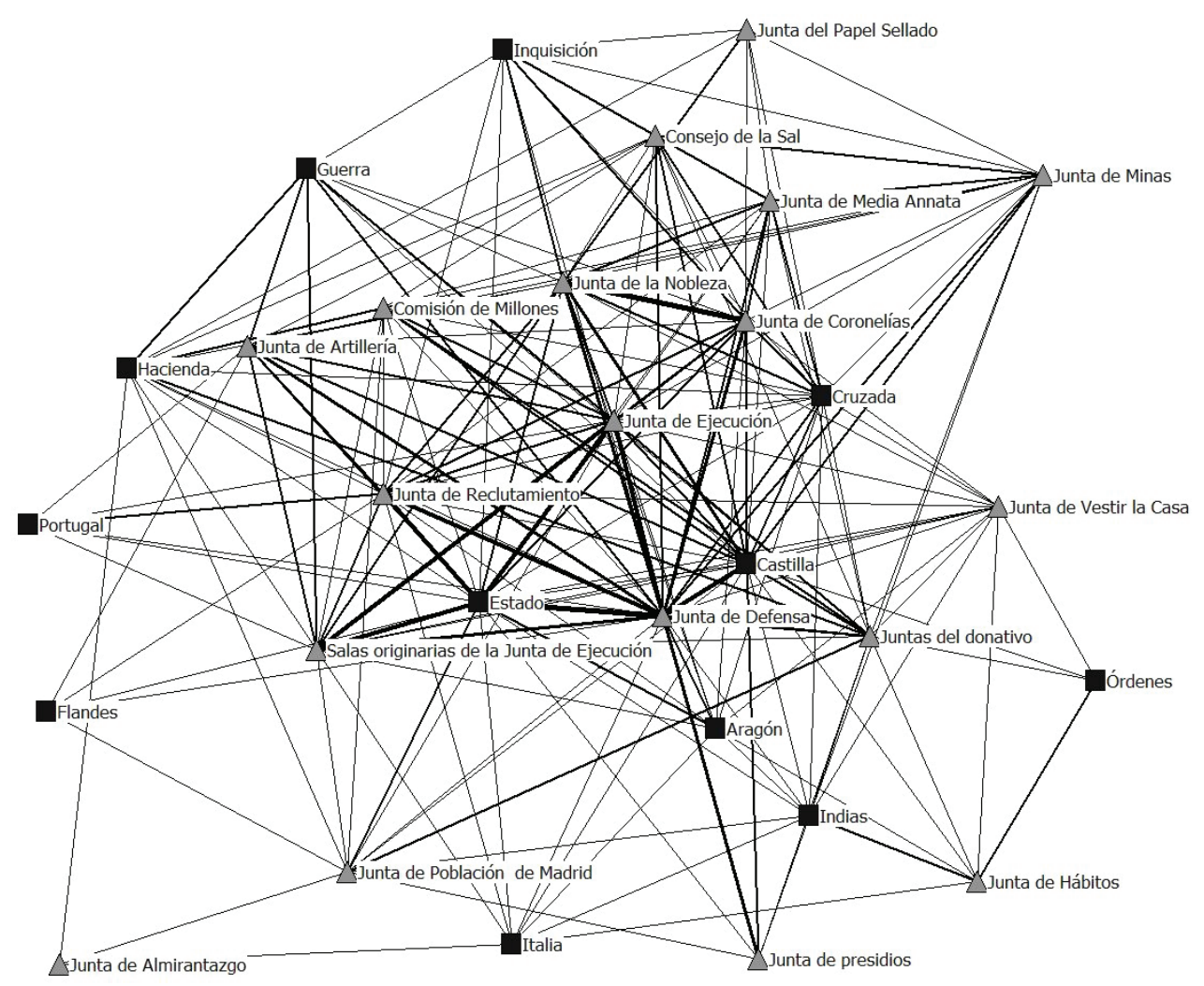

Son Juntas, y no Consejos, quienes ocupan la posición central, lo que denota la importancia de estas instituciones en el gobierno de Olivares. Los principales centros de poder administrativo, los Consejos, perdieron competencias a favor de unas nuevas instituciones, las Juntas, que replicaban su estructura y componentes, pero que mantenían un número mayor de relaciones entre sí que aquellos. Por otra parte carecían de una reglamentación asentada, lo que permitía una gran amplitud de movimientos.

El gráfico 3 nos muestra la posición de Juntas y Consejos, y su cercanía a los principales centros de decisión. Las relaciones entre estos dos tipos de instituciones, quedan patentes en un gráfico altamente interrelacionado con especial intensidad en el centro del mismo. Los Consejos, en 1635, ya habían sido prácticamente controlados por el Conde Duque de Olivares gracias a la introducción de hechuras y partidarios suyos. Resulta lógico que fuesen los miembros de la institución colegiada más po- 
derosa de la Monarquía, el Consejo de Estado, quienes formaran parte de las nuevas Juntas, asegurando así la cohesión del sistema. El mismo motivo origina que el Consejo de Castilla, el segundo en importancia, aparezca también en una posición central. Esta regla no se aplica al tercer Consejo con más relaciones, el de Cruzada, pues ostentaba mucho menos poder que los anteriores. La clave para entender la situación del Consejo de Cruzada en los gráficos hay que buscarla en sus miembros. A pesar de tener solo seis miembros acapararon mucho poder en otras instituciones, empezando por el confesor real e inquisidor general Fray Antonio de Sotomayor (129), a cuyos oficios en la administración se sumaba la influencia que tenía sobre Felipe IV y, por ende, en el entorno cortesano. También encontramos en el Consejo a dos camaristas de Castilla, Fernando Ramírez Fariñas (114) y José González (62), el principal hombre del Conde Duque en materias administrativas ${ }^{28}$. Los otros tres consejeros eran a su vez consejeros de Hacienda, Indias y Aragón respectivamente. La ausencia de consejeros exclusivos nos hace plantear la hipótesis de que el nombramiento de consejeros de Cruzada pudo emplearse como una forma de distinguir a los consejeros de otros Consejos.

Al hablar del sistema de Juntas debemos preguntarnos por los consejeros que no formaron parte de las ellas, así como por los miembros de éstas que no tenían plaza en uno de los Consejos de la monarquía. El número de consejeros que no pertenecieron a las Juntas fue elevado, 79 de un total de 121 . Es decir, que más del $65 \%$ de los consejeros se vieron excluidos de este nuevo circuito de poder que se estaba creando. No resulta aventurado decir que algunos lo fueron a modo de castigo, por no comulgar con las directrices políticas del Conde Duque. Sin embargo, tras varios años de privanza de Olivares, los Consejos habían sido progresivamente limpiados de opositores $y$, en cualquier caso, un $65 \%$ de oposición parece una cifra demasiado abultada para gobernar con comodidad durante tanto tiempo. Hemos de tener en cuenta para interpretar este dato que los consejeros llegaban a sus puestos tras una larga carrera en la administración, siendo la edad media de este colectivo bastante elevada para la época, lo que limitaba las actividades de muchos de ellos, poco deseosos de entrar a formar parte de nuevos organismos. Debemos mencionar también a los miembros del Consejo de Estado, nobles en su mayoría, y algunos incluso Grandes de España como el Duque de Alba, que no participaban en las tareas administrativas, y que por tanto entran dentro de este $65 \%$ que no participó en las Juntas.

Igualmente interesante resulta el caso contrario, el de los miembros de las Juntas que no procedían del sistema polisinodial, o al menos no de los puestos de consejeros. Algo más de un $38 \%$ de los miembros de las Juntas no tenían plaza de consejero, o lo que es lo mismo, 26 individuos de un total de 68 que formaban las Juntas estudiadas en este trabajo. Estos fueron oficiales con puestos en el entorno cortesano, o técnicos en alguno de los temas específicos que trataban las Juntas. Al no existir una regulación para los nombramientos de estos integrantes, se pudieron captar directamente hombres de valía fuera del sistema polisinodial. Qué duda cabe de que esta fue una nueva forma de crear clientelas por parte de quienes controlaban los nombramientos, con el Conde Duque de Olivares a la cabeza. Sin embargo, como se deduce de los

28 Ibidem, pp. 351-368. 
datos, no fueron un número demasiado elevado quienes dieron este salto en la carrera administrativa, por lo que debemos concluir que las Juntas estuvieron compuestas por una mayoría de consejeros que favorecieron la continuidad del sistema.

La participación en más de un Consejo estaba relacionada con la integración en más de una Junta. Puesto que los Consejos existían con anterioridad a la creación de las Juntas, podemos determinar que el valido eligió a consejeros, a los que su posición en la administración ya otorgaba una cierta independencia, en vez de crear nuevas hechuras. Para entender esta elección hemos de prestar atención a los nuevos órganos que se estaban implantando. Las Juntas restaban poder a los Consejos, lo que obviamente produjo la oposición de estos. Los Consejos de la Monarquía contaban con una extensa reglamentación que sancionaba sus competencias y obligaciones, a la que podían acogerse en caso de conflicto. En cambio, las Juntas carecían de ese soporte jurídico. Una de las formas de legitimar las Juntas fue la inclusión en ellas de destacados personajes de la administración y del entorno cortesano cuya influencia era utilizada para dar peso a estas instituciones. Pero sobre todo, las Juntas se formaron con miembros de los Consejos que poseían competencias en las materias que la Junta debía de tratar. Se solucionaban así parte de las trabas jurídicas pues, al ser los integrantes de la institución competentes en un determinado tema por estar en un Consejo, era difícil argumentar que la Junta creada para tratarlo no estaba capacitada. Por otro lado, se socavaban también las bases de la oposición al dividir al colectivo perjudicado, el de los consejeros, pues mientras que a unos les eran restadas competencias, otros veían consolidada su posición en las nuevas instituciones. Las Juntas fueron pues, una nueva forma de captar y fidelizar clientelas para el valido a la par que fueron utilizadas para aumentar la presión sobre sus opositores.

La elección de hombres con un gran peso en la administración dotaba a las Juntas del prestigio necesario para relacionarse con el resto de instituciones y particulares. Tradicionalmente se ha trabajado la relación del valido con los consejeros desde la posición dominante de éste gracias a su influencia sobre el monarca. Sin embargo, hemos de plantear la reciprocidad de esta relación, pues sin el apoyo de un nutrido grupo de consejeros, a Olivares le habría sido imposible introducir muchas de las novedades administrativas que se produjeron durante su valimiento. En esta relación bidireccional, el Conde Duque se sirvió de la posición que gozaban ciertos consejeros para dar peso a las nuevas instituciones a cambio de lo cual aumentó la influencia y riqueza de estos individuos multiplicando sus sueldos y empleos.

Del mismo modo que hemos realizado este primer análisis con las instituciones lo hemos hecho con sus miembros. En ese caso la matriz resultante vincula a los individuos que formaban parte de una misma institución. Como en el caso anterior, las celdas situadas en la diagonal se interpretan de forma diferente, pues corresponderían al mismo nombre en filas y columnas. Este número nos indica el número de instituciones de las que forma parte ese individuo, motivo por el cual no lo hemos incluido el gráfico resultante, ya que, además, la multiplicación de relaciones hace muy difícil su correcta visualización. Sin embargo, los elementos que podemos identificar tras el análisis de la representación gráfica están presentes en otras partes de este trabajo.

Cuando un gráfico se encuentra tan saturado de relaciones que no nos permite analizarlo visualmente, además del análisis de segundo nivel podemos emplear otras 
técnicas. Una de las más interesantes en este aspecto es la descomposición de la red en subgrupos. Existen diversas formas de acotar subgrupos, en este caso hemos empleado una de las más frecuentes, conocida como "k-cores", que se puede realizar, de forma automática, con la mayoría de los programas de software de redes como Netdraw $^{29}$. Podríamos simplemente averiguar qué personajes eran miembros de un mayor número de instituciones y contentarnos con esa aproximación, sin embargo ese sería un análisis demasiado plano que únicamente nos revelaría el número de nombramientos que recibieron. La descomposición en k-cores es un proceso con resultados similares pero mucho más ajustado a redes como ésta, pues no solo se valora el número de conexiones, sino que se tiene en cuenta la calidad con de los nodos que establecen una conexión. Se trata de que cuantas más conexiones tengan entre sí un grupo de nodos esos formarán parte de un k-core mayor. Esta técnica nos ha permitido identificar de forma clara al círculo más cercano de colaboradores del Conde Duque de Olivares.

\section{LAS HECHURAS DEL CONDE DUQUE}

Por tanto, a la luz de los resultados de los análisis que hemos planteado, se puede afirmar que formaron parte de la camarilla del Conde Duque los siguientes personajes: Manuel Acebedo Zúñiga y Fonseca, Conde de Monterrey; Francisco Antonio de Alarcón; Pedro de Arce; Carlos de Borja Barrete, Duque de Villanueva; Lorenzo de Cárdenas y Valda, Conde de la Puebla del Maestre; Francisco de Castelví; Juan de Castro y Castilla, Conde de Montalvo; Juan de Chaves, Vizconde de la Calzada; Antonio de Contreras; Bartolomé Espínola, Conde de Pezuela de las Torres; José González; García Avellaneda de Haro, Conde de Castrillo; Fernando de Llano y Valdés; Diego de Mesía y Guzmán, Marqués de Leganés; José de Nápoles; Ramiro Núñez de Guzmán, Duque de Medina de las Torres; Pedro Pacheco, Marqués de Castrofuerte; Lorenzo Ramírez de Prado; Fernando Ramírez Fariñas; Hernando de Salazar; Antonio de Sotomayor; Pedro Valle de la Cerda y Jerónimo de Villanueva.

Fueron estos veinticuatro hombres quienes controlaron gran parte de los resortes del Estado, asegurando la coordinación y el funcionamiento del sistema en las cuestiones de capital importancia. El papel de muchos de ellos es ya sobradamente conocido por la historiografía del periodo, sin embargo, creemos necesario identificar claramente a este grupo y llamar la atención sobre algunos de los nombres que permanecen ignorados por la historiografía del periodo. Partimos de la base de que el gobierno no recayó en un solo hombre, por mucho poder que pudiese acumular, sino

29 Un k-core es un grupo de actores en el cual todos están conectados, como mínimo, a un número (k) de miembros de ese grupo. Aplicando este concepto a la matriz de individuos e instituciones de la que partimos al principio del trabajo, hemos obtenido tres subgrupos. El primero, 1-core, engloba a todos los miembros de la red, pues todos tienen como mínimo una conexión. Según la definición que hemos dado, forman parte de 2-core los nodos que tienen al menos dos conexiones con otros miembros de la red. En 2-core, por tanto, ya se han eliminado los nodos con un único enlace y los que sólo se relacionan con nodos de una única relación. Sin embargo, el que nos interesa realmente es el más restrictivo, 3-core. En él únicamente aparecen aquellos nodos con tres o más vínculos con otros que a su vez cumplen la misma característica, por lo que la lista de individuos queda reducida a solo veinticuatro hombres. 
que fue compartido con una serie de personajes de su entorno. Pretendemos por ello, estudiar los mecanismos de gobierno que se emplearon en la cronología estudiada.

Entre los más conocidos de este grupo se encuentran los propios familiares del Conde Duque de Olivares: sus cuñados el Conde de Monterrey y el Conde de Castrillo, su yerno el Duque de Medina de las Torres y su primo el Marqués de Leganés. Este primer grupo se centra en el Consejo de Estado, como correspondía a sus cargos palaciegos y a la condición de nobles. Llama la atención el hecho de que los miembros del clan de los Guzmanes no presentan reparos a la hora de participar en Juntas y Consejos, tareas más propias de burócratas que de nobles. Aunque hemos de hacer una apreciación, y es que los parientes del Conde Duque generalmente formaron parte de las Juntas que anteriormente hemos catalogado como fundamentalmente políticas, siendo muy pocos los casos en los que estuvieron presentes en las Juntas técnicas.

Conviene reseñar el caso del Conde de Castrillo, que cursó estudios en Salamanca, donde llegó a ser rector como el propio Gaspar de Guzmán. Noble, con estudios y familiar del hombre más poderoso de la Monarquía, tenía todos los factores para realizar una carrera brillante, como así sucedió. García de Avellaneda y Haro ocupó la presidencia del Consejo de Indias desde 1632 hasta 1658, en que la abandonó para pasar a la presidencia del Consejo de Castilla, del que era miembro y camarista. Su trayectoria burocrática corrió pareja a la cortesana, llegando en 1640 a ser nombrado Mayordomo Mayor de Palacio, manteniendo una estrecha y cordial relación con la familia real. No tuvo problemas para sobrevivir políticamente a su valedor, contra el que parece que conspiró, y ocupar después cargos tan importantes como el de virrey de Nápoles ${ }^{30}$. A su participación en Juntas de todo tipo hemos de añadir que fue uno de los principales comisionados para las ventas de oficios públicos, especialmente los relacionados con Indias, durante el tiempo que presidió este Consejo ${ }^{31}$.

Miembros también de la nobleza titulada eran el Conde de la Puebla del Maestre y el aristócrata de origen portugués Carlos de Borja, Duque de Villahermosa y II Conde de Ficalho. Este, además de su posición en la Corte como gentilhombre de cámara, fue el principal entendido en todas las cuestiones referentes a Portugal como presidente del Consejo de Portugal.

Qué duda cabe de que el servicio al monarca en la alta administración y una fuerte vinculación con la facción gobernante constituían un trampolín excepcional desde el que acceder al estamento nobiliario. Durante sus años de privanza el Duque de Lerma había impulsado a algunas de sus hechuras hasta los ansiados títulos nobiliarios. Notorio es el caso del Marqués de Siete Iglesias, Rodrigo Calderón ${ }^{32}$, por la gran cantidad de poder que llegó a acumular. Parece que Olivares tomó nota de su antecesor, pues entre este grupo de estrechos colaboradores encontramos pocos nuevos titulados y siempre con meritorias carreras en la administración. Son los casos de Pedro Pacheco, que tituló como Marqués de Castrofuerte, Juan de Castro y Castilla, que lo hizo

\footnotetext{
30 Elliot, op. cit. (nota 6), p. 702.

31 Sanz TAPIA, A.: "Provisión, beneficio y venta de oficios americanos de Hacienda (1632-1700)", Revista Complutense de Historia de América, 37 (2011), p. 155.

32 Martínez Hernández, S.: Rodrigo Calderón. La sombra del valido. Privanza, favor y corrupción en la corte de Felipe III, Madrid, Marcial Pons Historia, 2009.
} 
como Conde de Montalvo, Bartolomé Espínola, con el título de Conde de Pezuela de las Torres, y Juan de Chaves, que obtuvo el título de Vizconde de la Calzada para sí y el de Conde de Santa Cruz para su hijo ${ }^{33}$.

El clero, también estuvo presente en la camarilla de Olivares. Nos estamos refiriendo al jesuita Hernando de Salazar, confesor del Conde-Duque, consejero de Inquisición, miembro de diversas Juntas, obispo de Málaga y posteriormente arzobispo de Charcas; a Fernando de Llano y Valdés, arzobispo de Granada y presidente del Consejo de Castilla; y al longevo dominico Fray Antonio de Sotomayor, confesor real, Inquisidor General y Comisario de Cruzada entre otros cargos. Estos tres personajes no sólo ocuparon algunos de los puestos más elevados de la administración, como el de presidente del Consejo de Castilla, sino que también ostentaron un elevado poder. En la corte fue sobradamente conocida la influencia del padre Salazar en asuntos de gobierno ${ }^{34}$, pero de forma específica en cuestiones de economía, siendo conocido como la "eminencia gris". El jesuita elaboró incluso un plan de reformas en un documento destinado al Conde Duque que coincide con muchas de las iniciativas políticas emprendidas por Olivares ${ }^{35}$. Entre esas propuestas estuvo la recomendación al valido de "que a los que tienen los primeros puestos los haga sus amigos tan intimos -si es posible-que se tengan por interesados en sus aciertos" ${ }^{36}$. No cabe duda de que el valido de Felipe IV siguió el consejo del eclesiástico. También fue notable la influencia que Antonio de Sotomayor, el confesor real, ejerció sobre Felipe IV, por lo que era una pieza fundamental a la hora de contar con el apoyo del monarca a una determinada política.

Los puestos en torno al monarca quedaron por tanto copados por partidarios de la facción olivarista. Los oficios de la Casa Real fueron el destino de varios miembros del clan Zúñiga-Guzmán, mientras que la conciencia del soberano era convenientemente guiada por el confesor real. El cerco a la corona quedó completado con los secretarios con el privilegio de despachar con el monarca, una de las vías más importantes de comunicación entre la administración y la cabeza del Estado, por lo que quienes las ocuparon debieron de ser olivaristas convencidos. Por un lado estaban los secretarios del Consejo de Estado, como Pedro de Arce, al frente de la Secretaría de Italia, territorio en el que estaba especializado. Como tal participó en la Junta de Defensa, en la poderosa Junta de Ejecución y en una de las salas que ocuparon su lugar cuando fue disuelta. Junto a él, en la secretaría de Aragón estuvo Jerónimo de Villanueva, que habiendo empezado como Protonotario de Aragón acabó siendo nombrado Secretario de Despacho. De él dice Elliott que fue "el hombre más poderoso de España después de Olivares" ${ }^{37}$. Esta posición tuvo su reflejo en los

33 FAYARD, op. cit. (nota 12), p. 163.

34 Elliot, op. cit. (nota 6), p. 173.

35 Respecto al padre Salazar remitimos a Dubet, A. y Negredo del Cerro, F.: "Le pouvoir de la grâce: le projet politique d'Hernando de Salazar, confesseur du comte-duc d'Olivares", Siècles. Cahiers du Centre d'Histoire «Espaces et Cultures», 13 (2001), pp. 19-28; NEgredo DEL CERro, F.: "La hacienda y la conciencia. Las propuestas del confesor del Conde Duque para el saneamiento de las finanzas reales (1625)", Cuadernos de Historia Moderna, 27 (2002), pp. 171-196; y Negredo del Cerro, F.: Política e Iglesia: los predicadores de Felipe IV, Madrid, Universidad Complutense, 2001, pp. 176-221.

36 Citado en Ibidem, p. 191.

37 ElliotT, op. cit. (nota 6), p. 470. 
nombramientos que fue acumulando como miembro de diversas Juntas y cargos de confianza del propio Felipe IV, como el de tesorero de los Gastos Secretos ${ }^{38}$. Tal fue la vinculación de Villanueva con el valido que ni siquiera los altos cargos que acumuló le permitieron sobrevivir políticamente a la caída de su protector. En 1643, ausente ya el Conde Duque de la Corte, la presión de sus enemigos produjo la reapertura de un proceso inquisitorial iniciado en 1628 del cual ya había sido absuelto con anterioridad. El proceso estuvo motivado por el escándalo protagonizado por las monjas del convento de San Plácido, fundado por Villanueva años antes, que habían llegado a tener revelaciones demoníacas ${ }^{39}$. Esto supuso el fin de su carrera política e incluso su encarcelamiento durante cuatro años.

Requisito para promocionar durante el periodo olivarista a los Consejos era la aquiescencia del valido, por lo que el control sobre los mismos fue haciéndose mayor con el tiempo. A la altura de 1635 Olivares llevaba ya suficientes años junto a Felipe IV en el poder como para haber limpiado de opositores los Consejos, especialmente los dos principales, Estado y Castilla ${ }^{40}$. Después del Consejo de Estado, en el que sirvieron un total de nueve individuos de este grupo de veinticuatro hombres, el Consejo con más miembros de la camarilla fue el de Castilla, con ocho. Todos los miembros de la Cámara de Castilla, el principal órgano en cuestión de nombramientos en la Monarquía Hispánica, empezando por el propio presidente del Consejo, Fernando de Llano y Valdés, fueron miembros de esta camarilla. Éste, como señalamos anteriormente, ostentaba el arzobispado de Granada y había sido nombrado para ocupar la presidencia en 1633. Fue el tercer presidente del Consejo de Castilla durante el valimiento de Olivares quien, tras las disputas con el primero de ellos, el cardenal Trejo ${ }^{41}$, conocía ya la importancia de contar con la colaboración del presidente del Consejo de Castilla.

Entre los demás consejeros de Castilla destaca la figura de José González, sin duda, junto al Protonotario, uno de los hombres más cercanos a Olivares, y cuyo favor le permitió realizar una rápida carrera en la administración que culminó en las instancias más altas, llegando a ser camarista de Castilla y consejero de Cruzada. Había empezado su carrera en la Chancillería de Valladolid, donde fue abogado de Olivares y desde donde promocionó a Alcalde de Casa y Corte, de allí a fiscal del Consejo de Castilla en 1628, y al año siguiente a consejero del mismo organismo. Resulta significativo que el ascenso al último peldaño, la Cámara de Castilla, no fuese por la vía ordinaria, mediante una consulta de la propia Cámara, sino mediante un decreto regio fechado en $1632^{42}$. No es necesario decir que el ascenso en cuatro años desde la fiscalía del Consejo de Castilla hasta la propia Cámara fue altamente irregular si lo comparamos con otros consejeros, lo que muestra claramente la mano del valido

\footnotetext{
38 SeIz Rodrigo, D.: La Disimulación honesta : los gastos secretos en el reinado de Felipe IV entre la razón de estado y la merced cortesana, Madrid, Endymion, 2010.

39 El proceso ha sido profundamente estudiado en PuYol BuIL, C.: Inquisición y politica en el reinado de Felipe IV: los procesos de Jerónimo de Villanueva y las monjas de San Plácido, 1628-1660, Consejo Superior de Investigaciones Científicas, 1993.

40 Amadori, A.: Política americana y dinámicas de poder durante el valimiento del Conde-Duque de Olivares, (1621-1643), Madrid, Universidad Complutense, 2011, pp. 484.

41 Elliott, op. cit. (nota 6), p. 442.

42 FAYARD, op. cit. (nota 27), pp. 351-368.
} 
detrás de todos estos nombramientos. Con estos títulos se justifica su participación en las Juntas de Minas, Donativo, Media Annata, Ejecución y Defensa, así como en el Consejo de la Sal y la Comisión de Millones. Fue, por tanto, uno de los individuos claves a la hora de controlar las nuevas instituciones, y muestra palmariamente los métodos del valido para promocionar a sus hechuras en el sistema polisinodial y en las Juntas.

La vía para promocionar a José González, el decreto regio, fue también empleada con algunos otros consejeros. Es el caso de Francisco Antonio de Alarcón, que fue nombrado consejero de Castilla en 1628, y que para la cronología que estudiamos ya era camarista de Castilla. Este madrileño se hizo cargo de asuntos militares, formando parte de la propia Junta de Defensa, de la Junta de Artillería y siendo incluso comisionado para reorganizar el ejército en Guipúzcoa en $1638^{43}$. También con un perfil relacionado con la milicia, formó parte del Consejo de Castilla Pedro Pacheco, que tituló como primer Marqués de Castrofuerte en 1627. Comisario General de la infantería de España, fue miembro del Consejo de Guerra y de todas las Juntas relacionadas con defensa, siendo comisionado para visitar diferentes plazas fuertes ${ }^{44}$. Posteriormente, fue nombrado miembro de de los Consejos de Indias y Estado ${ }^{45}$. Con este perfil no extraña tampoco que fuese nombrado gentilhombre de cámara del príncipe Baltasar Carlos y Mayordomo Mayor de la reina Isabel de Borbón. Lorenzo de Cárdenas Valda y Zárate, más conocido por ser el noveno Conde de la Puebla del Maestre, presenta similitudes con el Marqués de Castrofuerte, pues también acabó formando parte del entorno cortesano al ser nombrado Mayordomo de Felipe IV. La larga trayectoria como presidente de la Casa de Contratación, Asistente y Capitán General de Sevilla, consejero de Indias y de Estado culminó con el prestigioso nombramiento como Virrey de Nápoles, aunque no llegó a ejercer el cargo.

No todos los miembros de esta camarilla ascendieron gracias al valido, ya que muchos de ellos ya ocupaban puestos elevados de gobierno al comienzo del reinado. Uno de ellos fue el camarista de Castilla Fernando Ramírez Fariñas, que ocupaba plaza de consejero desde 1616, y que colaboró de forma muy activa con la facción olivarista, realizando visitas o recaudando el donativo ${ }^{46}$ hasta su muerte en $1638^{47}$. En el caso de Antonio de Contreras, se cruzan dos factores que le ayudaron a conseguir en 1630 el nombramiento como camarista. Por un lado, su primo, Francisco de Contreras, presidente del Consejo de Castilla entre 1621 y 1627, y por el otro su amistad con José González ${ }^{48}$.

43 FAYARD, op. cit. (nota 12), pp. 86, 112.

44 Gil Sanjuán, J.: "Visita a Málaga de don Pedro Pacheco, comisario militar de Felipe IV", Baética: Estudios de arte, geografia e historia, 25 (2003), pp. 527-546.

45 Barrios, F.: El Consejo de Estado de la Monarquía Española, Madrid, Consejo de Estado, 1984, pp. 372.

46 Lanza García, R.: "El donativo de 1629 en el distrito de Fernando Ramírez Fariñas", en IX Congreso Internacional de la Asociación Española de Historia Económica, Murcia, 2008. Pérez JuAN, J. A.: "La visita de Ramírez Fariña a la Audiencia de Sevilla (1623-1632)", Historia, instituciones, documentos, 29 (2002), pp. 357-396.

47 FAYARD, op. cit. (nota 12), p. 393.

48 Ibidem, pp. 239 y 448. 
Aunque Estado y Castilla fueron los Consejos donde se concentraron los más estrechos colaboradores de Olivares, se distribuyeron también por el resto de Consejos, ocupando plazas de consejeros y también de presidentes. A los anteriormente analizados debemos añadir a Juan de Chaves y Mendoza, camarista de Castilla, pero también presidente del Consejo de Órdenes. A través de este personaje se establecía una vía de comunicación entre ese Consejo, más alejado de los círculos de poder, y el núcleo dónde se tomaban las decisiones más importantes de la Monarquía. Lorenzo Ramírez de Prado, conocido bibliófilo y humanista de la época, era consejero de Indias y de Cruzada; Pedro Valle de la Cerda lo era de Hacienda y Cruzada; y Francisco de Castelví lo fue de Aragón y Cruzada. Se repetía por tanto el esquema de pertenencia a un Consejo con unas competencias amplias y a otro de ámbito más restringido, que favorecía la integración de las instituciones más aisladas.

Los parientes del Conde Duque mantenían entre sí unas relaciones basadas en el parentesco, pero podemos comprobar cómo el resto de esta camarilla también estableció relaciones de amistad e incluso también de parentesco. Jerónimo de Villanueva, Protonotario de Aragón y mano derecha de Olivares, tenía como cuñado a Pedro Valle de la Cerda. Pero el mejor ejemplo, es el de Lorenzo Ramírez de Prado, que casó con Lorenza de Cárdenas, hermana del Conde de la Puebla del Maestre, y tuvo por padrino de boda a Juan de Chaves ${ }^{49}$. Además, a su muerte dejó obras de arte a Antonio de Contreras y al conde de la Puebla ${ }^{50}$, herederos a los que también podemos encontrar en la camarilla. Este tipo de relaciones que se dieron entre muchos de los personajes que formaron parte de la alta administración de la Monarquía, demuestran la fuerza de los vínculos existentes entre ellos.

En el grupo que hemos identificado, abundan los nobles, burócratas de carrera, y algunos religiosos. Únicamente encontramos a un personaje que se sale de estos perfiles: Bartolomé Espínola. Este consejero de Guerra y Hacienda de origen genovés no procedía de los peldaños inferiores de la administración, sino que, como muchos de sus compatriotas, se dedicaba a los negocios y al mundo de las finanzas. Nombrado Factor General en $1627^{51}$, Bartolomé fue desde entonces quien negoció muchos de los asientos de la Monarquía y llevó a cabo otros negocios y arbitrios como la venta de escribanías de millones ${ }^{52}$. Por estos y otros servicios a la corona fue recompensado en 1642 con el título de Conde de Pezuela de las Torres. Sus relaciones con financieros genoveses, incluyendo a su propio hermano, fueron de utilidad para la Monarquía en un momento en el que era necesario movilizar todos los recursos disponibles.

A pesar de que los miembros del grupo estudiado pertenecían a instituciones de diversa naturaleza y temática, no ha de interpretarse como que los mismos personajes formaban parte de todas las Juntas independientemente de la temática que estas tuvieran. Debemos señalar que los consejeros formaban parte de Juntas con temáticas afines, de modo que no se perdiese su especialización en determinadas materias. José de Nápoles fue, por ejemplo, ministro indispensable para todas las cuestiones que afectaban a Italia; Jerónimo de Villanueva para Aragón; y el Duque de Villahermosa

\footnotetext{
49 Entrambasaguas, J.: Una familia de ingenios: los Ramírez de Prado, Madrid, CSIC, 1943, p. 71.

50 FAYARD, op. cit. (nota 12), p. 459.

51 Álvarez Nogal, op. cit. (nota 26).

52 Archivo General de Simancas (AGS), Consejo y Juntas de Hacienda, Leg. 752.
} 
para Portugal. Francisco Antonio de Alarcón y el Marqués de Castrofuerte lo eran en temas militares, Hernando de Salazar y Bartolomé Espínola en temas hacendísticos. También hemos constatado la diferenciación entre los miembros de las Juntas de carácter político, que solían ser los parientes del Conde Duque, y los de las Juntas de carácter técnico, que generalmente estaban formadas por burócratas de carrera que tenían plaza en los Consejos de Castilla, Hacienda o Indias.

A todos estos nombres habría que añadir algunos que, por las características de los datos y del método que hemos empleado, han quedado excluidos del grupo al que hemos identificado como camarilla. El motivo fundamental es que nos hemos basado en la multiplicidad de cargos, no en las diferentes cotas de poder de los cargos. Tampoco hemos podido abarcar todas las Juntas del periodo, pues de muchas de ellas no se ha conservado documentación alguna. El último elemento que produce distorsión en los resultados son los personajes que, sin estar directamente vinculados con la administración, ejercieron una notable influencia en virtud de su posición en la Corte. Así, por ejemplo, debemos hacer referencia a hombres como el secretario particular del Conde Duque, Antonio Carnero y su ayudante Jerónimo de Lezama ${ }^{53}$, el secretario de Estado de Flandes, Andrés de Rozas, y Fernando Ruiz de Contreras, secretario de la Junta de Ejecución y del Consejo de Guerra ${ }^{54}$. Otro personaje excluido de este grupo que ocupó un puesto de especial relevancia en la Corte y en la alta administración fue Antonio de Camporredondo y Río, gobernador del Consejo de Hacienda. En las obras generales sobre el período ha sido frecuentemente citado como miembro de multitud de Juntas ${ }^{55}$, no obstante, hemos podido constatar su participación en Juntas extraordinarias de temática económica muy vinculadas al propio Consejo de Hacienda que, por su propio carácter extraordinario, han quedado fuera de la muestra utilizada en esta investigación.

A pesar de estas deficiencias, creemos que el análisis de redes y la teoría de grafos son herramientas que nos pueden desvelar procesos y relaciones que de otra forma serían indetectables. Para su uso, han de ser requisitos indispensables la combinación con otras metodologías y el exhaustivo conocimiento de la bibliografía y las fuentes documentales. La aplicación combinada de estas técnicas nos ha permitido ver el sistema de Juntas de una forma diferente, entendiéndolo como un sistema continuista con la estructura polisinodial, pues los Consejos mantuvieron su posición pese a todo, pero con una serie de nuevas características, acordes a las exigencias del gobierno del Conde Duque de Olivares.

Este grupo que hemos identificado como miembros de la camarilla dependió del valido para poder formar parte de las Juntas, una forma de acumular poder y relaciones personales, que al mismo tiempo suponían nuevas oportunidades de enriquecimiento. El peso de la figura de Gaspar de Guzmán en la historiografía ha eclipsado, en buena medida, el papel de estos hombres, sin los cuales resulta imposible concebir esta etapa de gobierno. Conocemos bastante sobre los hombres más principales y sobre los propios familiares del Conde Duque. Sin embargo, excluyendo las referencias

53 Elliot, op. cit. (nota 6), p. 326.

54 Ibidem, p. 614.

55 Fayard y Baltar han repetido el dato dado en Domínguez Ortiz, A.: Política y Hacienda de Felipe IV, Madrid, Editorial de Derecho Financiero, 1960, p. 185. 
que hemos dado, apenas se menciona la importancia en el gobierno de hombres como Francisco Antonio de Alarcón, Francisco de Castelví, Juan de Castro y Castilla, Juan de Chaves, Antonio de Contreras, José de Nápoles, Pedro Pacheco, Fernando Ramírez Fariñas, Pedro Valle de la Cerda o Pedro de Arce, hombres que fueron a todas luces fundamentales durante el valimiento de Olivares.

El Conde Duque llevó el timón de la nave del Estado durante más de dos décadas, pero no menos importante es conocer a quienes ejercieron de remeros durante ese tiempo. Este trabajo es un ejemplo de las posibilidades del análisis de redes como metodología, pues nos permite abrir nuevas interpretaciones y obtener nuevas conclusiones a partir de los datos ya conocidos.

\section{ANEXO}

\section{INSTITUCIONES Y LEYENDA}

Consejos:

(E) Estado

(C) Castilla

(I) Indias

(G) Guerra

(H) Hacienda

(IT) Italia

(A) Aragón

(IN) Inquisición

(O) Órdenes

(P) Portugal

(CR) Cruzada

(F) Flandes

$\underline{\text { Juntas: }}$

1. Junta de Minas

2. Junta de Población de Madrid

3. Junta de Almirantazgo

4. Juntas del Donativo

5. Junta de Media Annata

6. Consejo de la Sal

7. Junta de Ejecución de las prevenciones de la Defensa de los Reinos / Junta de Defensa

8. Junta de Presidios

9. Junta de Coronelías

10. Junta de Artillería

11. Junta de Hábitos

12. Junta de Reclutamiento 
13.Junta del Papel Sellado

14. Junta de Ejecución

15. Salas originarias de la disuelta Junta de Ejecución

16. Comisión de Millones

17. Junta de la Nobleza

18. Junta de Vestir la Casa

\section{CONSEJEROS Y MIEMBROS DE LAS JUNTAS}

1. Acebedo Zúñiga y Fonseca, Manuel Monterrey [Conde]: 15; E; IT.

2. Aguirre, Iñigo: IT.

3. Alarcón, Francisco: C.

4. Alarcón, Francisco Antonio: 1; 6; 7; 10; C.

5. Alba [Duque]: E.

6. Alcalá [Duque]: E.

7. Alfaro, Francisco: $\mathrm{H}$.

8. Almeyda de, Cid: 12 ; P.

9. Altamirano, Diego: O.

10. Álvarez de Bohórquez y Girón, Antonio Trujillos [Marqués]: H.

11. Anaya de, Bartolomé: 7; G.

12. Andía Irrazabal de, Francisco Valparaíso [Marqués]: 7; G.

13. Arce, Pedro: 7; 14; 15; E.

14. Avellaneda Haro de, García Castrillo [Conde]: 2; 4; 7; 8; E; C; I.

15. Bautista de la Rea, Juan: 4; H.

16. Benavente y Benavides, Cristóbal: 15 .

17. Borja [Cardenal]: E.

18. Borja Barrete y Aragón de, Carlos Villahermosa [Duque]: $7 ; 10 ; 12 ; 14 ; 15 ; \mathrm{E} ; \mathrm{P}$

19. Bracamonte, Gaspar Peñaranda [Conde]: C.

20. Brancacho, Lelio (Fr.): E.

21. Brancha, Ferrante: IT.

22. Brizuela de, Diego: 3 .

23. Bullón, Sancho: $\mathrm{H}$.

24. Cabrera y Bobadilla, Luis Jerónimo Chinchón [Conde]: 4; E.

25. Cadereyta [Marqués]: G.

26. Camporredondo de, Antonio: $16 ; \mathrm{H}$.

27. Cano, Domingo (Fr.): 5.

28. Cárdenas de, Diego: 11; I.

29. Cárdenas y Valda de, Lorenzo Puebla [Conde]: 7; 12; E; H.

30. Cardona [Duque]: E.

31. Carnero, Antonio: 3; IT.

32. Carrillo de Mendoza y Pimentel, Diego: E.

33. Carvajal de, Miguel: 17; C.

34. Castelví de, Francisco: 7; A; CR. 
35. Castilla de, Juan: 7; 8 .

36. Castillo Alvarado, Juan: 13.

37. Castillo del, Francisco: $\mathrm{H}$.

38. Castillo del, Juan: $\mathrm{H}$.

39. Castro y Castilla de, Juan Montalvo [Conde]: 4; G; H.

40. Caymo, Jerónimo: 2.

41. Cerralvo [Marqués]: G.

42. Chaves de, Juan: $4 ; 11 ; 18 ; \mathrm{C} ; \mathrm{O}$.

43. Chumacero de, Antonio: 8; C.

44. Chumacero de, Juan: C.

45. Cid, Nicolás: 14.

46. Coloma, Carlos Espinar [Marqués]: 14; E.

47. Coloma, Pedro: G.

48. Conca [Príncipe]: G.

49. Contreras de, Antonio: 12; 16; C.

50. Contreras, Diego: 4.

51. Cornejo, Rafael: $\mathrm{H}$.

52. Crois, Juan Solre [Conde]: 2; F

53. Dávila y Zúñiga, Antonio Mirabel [Marqués]: E.

54. Doria, Horacio: 4.

55. Enríquez, Juan: IT.

56. Espínola, Bartolomé: 15; G; H.

57. Fernández de la Cueva, Francisco Alburquere [Duque]: E; A.

58. Fernández Puertocarrero, Juan Dionisio: IN.

59. Fuentes [Marqués]: G.

60. Gavarela de, Mos: F

61. Gómez de la Asperilla, Francisco: $\mathrm{H}$.

62. González, José: $1 ; 4 ; 5 ; 6 ; 7 ; 14 ; 16 ;$ C; CR.

63. Gudiel y Peralta, Luis: 18 .

64. Guillén de la Carrera, Alonso: C; IT.

65. Guzmán de, Gaspar Olivares [Conde-duque]: 2; 4; 14; 15; 16; E.

66. Haro de, Pedro: G.

67. Henríquez, Antonio (Fr.): 5.

68. Herrera de, Pedro: $\mathrm{H}$.

69. Hinojosa de, Manuel: $\mathrm{H}$.

70. Hojeda, Fernando: H.

71. Hurtado de Mendoza, Juan Hinojosa [Marqués]: 2.

72. Ibarra de, Carlos: G.

73. Ipeñarrieta de Miguel: $16 ; \mathrm{H}$.

74. Jirón, Juan: O.

75. Lezama de, Pedro: $\mathrm{H}$.

76. Llano y Valdés de, Fernando: 7; 9; 17; C.

77. López Madera, Gregorio: 1; C.

78. Luna de, Antonio: 11; O.

79. Magarola, Juan: A. 
80. Mascareñas, Francisco: P.

81. Medina Sidonia [Duque]: E.

82. Melo de, Francisco: $P$.

83. Mendoza de, Juan: $O$.

84. Mendoza, Antonio: IN.

85. Mendoza, Juan: 2.

86. Mesía de Guzmán, Diego Leganés [Marqués]: 7; 10; 12; E; F

87. Mesía, Diego: 3 .

88. Monroy de, Sancho Castañeda [Marqués]: 15.

89. Montejasso [Marqués]: G.

90. Morales de, Lope: O.

91. Morquecho, Bartolomé: I.

92. Mortantes de, Agustín: A.

93. Mota da, Mendo: 2.

94. Mota de la, Gilimón: 2; 3; H.

95. Muñoz Escobar, Juan: 6 ; $\mathrm{H}$.

96. Nápoles de, José: 7; 12; IT.

97. Neila, Pedro: IT.

98. Neoburgo [Duque]: E.

99. Núñez de Guzmán, Ramiro Medina de las Torres [Duque]: 11; I; IT; A.

100. Oces de, Lope: G.

101. Oleaga, Sebastián: H.

102. Oquendo de, Antonio: G.

103. Ortiz de Sotomayor, Gabriel: IN.

104. Pacheco, Pedro Castrofuerte [Marqués]: 7; 9; 10; 14; 15; 17; C; G.

105. Palafox y Mendoza de, Juan: 11; I.

106. Pardo, Juan: I.

107. Paredes, Luis: C.

108. Pedroso, Juan: 3.

109. Pérez Valenzuela, Gonzalo: 1.

110. Pimentel, Enrique Cuenca (Obispo): E.

111. Pizarro, Fernando: 9; 17.

112. Porras de, Felipe: H.

113. Ramírez de Prado, Lorenzo: 18; I; CR.

114. Ramírez Fariñas, Fernando: 6; 9; 17; C; CR.

115. Riaño de, Diego: C.

116. Ribera, Bernardo: 7; 8.

117. Rojas de, Luis: G.

118. Rosa de, Pedro: F

119. Rozas de, Andrés: E.

120. Ruiz de Contreras, Fernando: 14 ; G.

121. Salazar de, Hernando: $1 ;$; 9 ; 13; 17; IN.

122. Salcedo de, Diego: G.

123. San Agustín de, Juan (Fr.): 5.

124. San Vítores, Jerónimo: 7; 8. 
125. Santa Cruz [Marqués]: E.

126. Santos de San Pedro, Miguel: 5.

127. Silva, Felipe: 10; G.

128. Solórzano y Pereira, Juan: 1; I.

129. Sotomayor de, Antonio (Fr.): 5; 7; 9; 12; 17; E; IN; CR.

130. Spínola, Felipe Balbases [Marqués]: 14.

131. Tursis [Duque]: E.

132. Valdés, Antonio: C.

133. Valle de la Cerda, Pedro: $13 ; 17 ; \mathrm{H} ; \mathrm{CR}$.

134. Vasconcelos de, Manuel: P.

135. Vayetola, Matías: A.

136. Vélez de Guevara y Tassis, Íñigo Oñate [Conde]: O.

137. Vico, Francisco: A.

138. Villafranca [Marqués]: E.

139. Villanueva de, Jerónimo: $7 ; 9 ; 12 ; 14 ; 15 ; 17 ; 18$; E; A.

140. Villanueva de, Pedro: 14.

141. Villaseñor de, Fernando: I.

142. Vivanco de, Pedro: I.

143. Zapata, Francisco: G; IN.

144. Zeballos de, Diego: O.

145. Zembrana de, Sebastián: O.

146. Zisternes, Melchor: A.

147. Zúñiga y Avellaneda, Juan Miranda [Conde]: 15. 